\title{
Cisplatin and Gemcitabine With Either Vinorelbine or Paclitaxel in the Treatment of Carcinomas of Unknown Primary Site
}

\author{
Results of an Italian Multicenter, Randomized, Phase II Study
}

\author{
Sergio Palmeri, MD ${ }^{1}$ \\ Vito Lorusso, $\mathrm{MD}^{2}$ \\ Laura Palmeri, MD ${ }^{1}$ \\ Marina Vaglica, MD ${ }^{1}$ \\ Camillo Porta, MD $^{3}$ \\ Rolando Nortilli, MD ${ }^{4}$ \\ Francesco Ferraú, MD $^{5}$ \\ Giuseppe Comella, ${ }^{6}{ }^{6}$ \\ Bruno Massidda, MD $^{7}$ \\ Marco Danova, MD $^{3}$ \\ for the Carcinomas of Unknown \\ Primary Italian Study Group \\ ${ }^{1}$ Department of Oncology, Division of Medical \\ Oncology, University of Palermo, Palermo, Italy. \\ ${ }^{2}$ Medical Oncology, Cancer Institute of Bari, Bari, Italy. \\ ${ }^{3}$ Medical Oncology, Scientific Institute for Research \\ and Cure, San Matteo, Pavia, Italy. \\ ${ }^{4}$ Division of Medical Oncology, University of \\ Verona, Verona, Italy. \\ ${ }^{5}$ Medical Oncology, Taormina Hospital, Taormina, Italy. \\ ${ }^{6}$ Medical Oncology A, Tumor Institute Fondazione \\ Pascale, Naples, Italy. \\ ${ }^{7}$ Division of Medical Oncology, University of \\ Cagliari, Cagliari, Italy.
}

Partially funded by ex $60 \%$ research funds of the University of Palermo.

We thank Mr. Valerio Gioé for revising the English article.

The following clinicians actively participated in the study and are considered coauthors: Gianfranco Filippelli, MD (Department of Medical Oncology, Paola Hospital, Paola, Italy); Maria Catena Macaluso, MD

BACKGROUND. To date, the standard treatment for patients who have carcinoma of unknown primary site has not been established.

METHODS. In this randomized Phase II study, 66 previously untreated patients (33 patients per arm) with carcinomas of unknown primary site received cisplatin $\left(35 \mathrm{mg} / \mathrm{m}^{2}\right)$ and gemcitabine $\left(1000 \mathrm{mg} / \mathrm{m}^{2}\right)$ with either paclitaxel $\left(70 \mathrm{mg} / \mathrm{m}^{2}\right)$ or vinorelbine $\left(25 \mathrm{mg} / \mathrm{m}^{2}\right)$, and all drugs were administered intravenously on Days 1 and 8 of a 21 -day cycle. Twenty-nine patients (44\%) presented with $\geq 2$ involved sites. The pathologic diagnosis was mainly adenocarcinoma (48 patients; $72.7 \%$ ) and squamous carcinoma (7 patients; $10.6 \%$ ).

RESULTS. In the first arm, 16 patients (48.5\%) experienced an objective response, and 9 patients (27.2\%) had disease stabilization. In the vinorelbine-containing arm, 14 patients (42.3\%) experienced an objective response, and 8 patients $(24.2 \%)$ had disease stabilization. The median response duration and the median time to progression were similar in both treatment arms; the median overall survival was 9.6 months (95\% confidence interval, 7.11-12.09 months) for patients who received the cisplatin/gemcitabine/paclitaxel regimen and 13.6 months (95\% confidence interval, 6.61-20.59 months) for patients who received the vinorelbine combination. Grade 3 and 4 toxicities were more frequent in the paclitaxel-containing arm.

CONCLUSIONS. Both combinations satisfied the 2-step design, demonstrating antitumor activity without relevant differences in response rates or response duration; however, the vinorelbine-containing regimen yielded superior results both in terms of overall survival (13.6 months vs 9.6 months) and in terms of treatment tolerability. Therefore, according to a pick the winner attitude, the combination of cisplatin/ gemcitabine/vinorelbine may be considered in the design of future randomized, Phase III trials for patients with carcinomas of unknown primary site. Cancer 2006;107:2898-905. (C) 2006 American Cancer Society.

KEYWORDS: carcinoma, unknown primary, chemotherapy, cisplatin, gemcitabine, vinorelbine, paclitaxel.

(Department of Oncology, Division of Medical Oncology, University of Palermo, Palermo, Italy); Anna Russo, MD (Department of Oncology, Division of Medical Oncology, University of Palermo, Palermo, Italy); Paolo Tralongo, MD (Medical Oncology, Avola, Syracuse, Italy); Giuseppe De Cataldis, MD (Oncology, General Hospital of Procida, Salerno, Italy); Annamaria Bochicchio, MD (Centro Rif. Oncologico Basilicata-DH Oncologico, Rionero in Vulture, Italy); Raffaella Berté Raffaella, MD (Department of Medicine I-Oncohematology, Azienda Civil Hospital, Piacenza, Italy); L.
Majorino (Medical Oncology, San Gennaro Hospital, Naples, Italy); and Vincenzo Accurso, MD (Department of Oncology, Division of Medical Oncology, University of Palermo, Palermo, Italy).

Address for reprints: Sergio Palmeri, MD, Division of Medical Oncology, Department of Oncology, University of Palermo, Via del Vespro 129, 90127 Palermo, Italy; Fax: (011) 39 0916552508; E-mail: sergiopalmeri@ alice.it 
C arcinomas of unknown primary site (CUP) represent a distinct but heterogeneous group of metastatic tumors, accounting for $2.3 \%$ to $4.2 \%$ of all cancers $^{1}$ in which the primary site of origin cannot be detected during pretreatment evaluation. ${ }^{2}$ Despite significant improvement in many diagnostic techniques, eg, tumor imaging and pathologic evaluation of tissue samples, CUP represents an extremely challenging clinical entity in terms of treatment. Indeed, the real impact of chemotherapy on the natural history of CUP remains to be determined, and the median survival of patients with CUP rarely exceeds 12 months. ${ }^{3}$ Although cisplatin-based regimens have yielded better response rates (usually in the range from $23 \%$ to $39 \%$ ), median survival results remain unsatisfactory, ranging from 5 months to 9 months, ${ }^{4}$ and no standard treatment has been established to date. ${ }^{5}$

The recent availability of newer, broadly active chemotherapeutic agents (eg, gemcitabine, vinorelbine, and the taxanes, among others) has created new options in the therapeutic strategy for patients with CUP. Here, we report the results of a multicenter, randomized, Phase II trial evaluating the efficacy and toxicity profile of the combination of cisplatin (Pfizer Pharmaceuticals, New York, NY) and gemcitabine (EliLilly, Indianapolis, IN) combined either with vinorelbine (PierreFabre Medicament, Castres Cedex, France) or with paclitaxel (Bristol-Myers Squibb, Princeton, NJ) for the treatment of CUP.

\section{MATERIALS AND METHODS Diagnostic Workup}

Patients with histologically (or cytologically) documented, metastatic carcinoma were considered eligible for the current study when the following diagnostic procedures did not allow a primary origin to be identified: complete history and thorough physical examination, blood chemistry (including, in men, serum tumor marker for prostate-specific antigen [PSA], $\alpha$-fetoprotein, and $\beta$-human chorionic gonadotropin), urinalysis, mammography in women, thoracic and abdominopelvic computed tomography scans, bone scan, and symptom- or sign-oriented imaging or endoscopic studies. Patients were enrolled if light microscopic pathologic analysis showed a well or poorly differentiated adenocarcinoma, a poorly differentiated carcinoma, or any squamous cell carcinomas. Specific pathologic analyses were mandatory to rule out lymphomas (ie, staining for leukocyte common antigen), malignant melanoma (ie, staining for both S100 and HMB45), extragonadal germ cell neoplasms (ie, staining for both $\alpha$-fetoprotein and $\beta$-human chorionic gonadotropin), sarcomas (ie. staining for cytokeratins and vimentin), neuroendocrine tumors (ie, staining for chromogranin and synaptophysin), and prostatic adenocarcinomas in men (ie, staining for PSA).

\section{PATIENTS}

Along with a histologically (or cytologically) confirmed diagnosis of CUP, eligibility criteria included measurable disease, an Eastern Cooperative Oncology Group (ECOG) performance status (PS) $\leq 2$, age between $\geq 18$ years and $\leq 75$ years, a life expectancy $\geq 12$ weeks, and written informed consent obtained according to the institutional requirements.

Patients also were required to have adequate organ function, which was defined by the following parameters: white blood cells $\geq 3000 / \mu \mathrm{L}$, platelets $\geq 100,000 / \mu \mathrm{L}$, hemoglobin $\geq 9.5 \mathrm{~g} / \mathrm{dL}$, bilirubin $<1.5 \mathrm{mg} / \mathrm{dL}$, aspartate and alanine aminotransferase levels $<3$ times the upper limit of normal, and creatinine $<2.0 \mathrm{mg} / \mathrm{dL}$. The presence of significant comorbidities, including uncontrolled diabetes, active systemic infections, a history of severe coronary artery disease or myocardial infarction within the last 6 months, psychiatric conditions, the presence of central nervous system metastases, a history of other cancers within the previous 5 years (except localized non-melanomatous skin cancer or wellmanaged in situ uterine carcinoma), and pregnancy or lactation, were considered exclusion criteria. Patient subgroups that were suitable for well-defined treatments (ie, women with adenocarcinoma involving axillary lymph nodes as the only site of disease, women with papillary serous carcinoma of the peritoneum, patients with squamous cell carcinoma that involved either cervical or inguinal lymph nodes only, patients with poorly differentiated carcinomas that suggested germinal tumors and with elevated levels of $\beta$-human chorionic gonadotropin and/or $\alpha$-fetoprotein, and patients with carcinoma that involved a single, potentially resectable site) also were excluded from enrolment.

\section{Treatment Schedule}

In the first treatment arm (cisplatin, gemcitabine and vinorelbine $[\mathrm{CGV}])$, vinorelbine $\left(25 \mathrm{mg} / \mathrm{m}^{2}\right)$ was added to a cisplatin $\left(35 \mathrm{mg} / \mathrm{m}^{2}\right)$ and gemcitabine $(1000 \mathrm{mg} /$ $\mathrm{m}^{2}$ ) combination and all drugs were administered intravenously on Days 1 and 8 of a 21-day cycle. Gemcitabine was administered over 30 minutes before cisplatin.

In the second treatment arm (cisplatin, gemcitabine, and paclitaxel [CGT]), paclitaxel $\left(70 \mathrm{mg} / \mathrm{m}^{2}\right)$ given as a 1-hour intravenous infusion, replaced the vinorelbine. Cisplatin and gemcitabine were delivered as specified above for the CGV arm.

In the CGT arm, all patients received premedication with steroids and antihistamine drugs according 
to the practice of each center. Antiemetic treatment was provided at each researcher's discretion.

A minimum of 3 cycles was required before the first tumor response assessment; then, a complete reevaluation was performed every 3 cycles of chemotherapy employing the same staging procedures that identified the metastatic sites of disease at baseline. The study treatments were administered mainly on an outpatient basis.

\section{Assessment of Response and Toxicity}

Complete response (CR), partial response (PR), stable disease (SD), and progressive disease (PD) were assessed according to World Health Organization criteria. $^{6}$ A CR was defined as complete regression of all lesions and signs and symptoms of disease. A PR was defined as a reduction $\geq 50 \%$ in the sum of the product of the 2 greatest dimensions of all measurable lesions, including partial healing of lytic lesions or reduction in the number of the uptake areas, that lasted $\geq 2$ months. SD was considered a reduction $<50 \%$ or an increase $<25 \%$ in the sum of the product of the same 2 greatest dimensions of measurable lesions that lasted $\geq 3$ months. PD was defined as an increase $>25 \%$ in the sum of the product of the 2 greatest dimensions of all measurable lesions and/or the appearance of new lesions. Chemotherapy-related toxic effects were recorded according to the National Cancer Institute's Common Toxicity Criteria, version $3.0,{ }^{7}$ at every cycle and at the end of treatment.

\section{Statistical Considerations}

The primary endpoint of this randomized Phase II study was the evaluation of the overall response rate (ORR); secondary objectives were the analysis of the toxicity profile, the time to progression (TTP), the duration of response, the overall survival (OS) for the 2 treatment arms, the correlation between disease characteristics (extension, sites of disease, etc), and clinical and/or pathologic parameters. A noncomparative analysis of these parameters in both treatment arms was performed to select the best regimen according to a pick the winner attitude. For this randomized Phase II study, the sample size was calculated by applying a Simon 2-step minimax design. ${ }^{8}$

Considering a $40 \%$ ORR clinically important with a type- 1 error of $5 \%$ and a type- 2 error of $20 \%, 18$ patients in each treatment arm were required for initial treatment. If $\geq 5$ of the first 18 patients obtained an objective response, then accrual would continue until the final enrolment of a total of 33 patients per arm. The TTP was calculated the date chemotherapy was started until there was clinical and/or radiologic evidence of $\mathrm{PD}$; OS was calculated from the date chemotherapy was started until the date of death or last documented follow-up. All data concerning TTP and OS were analyzed according to the Kaplan-Meier method $^{9}$ by using the SPSS statistical software package. Finally, to determine whether some clinical parameters (patient age, ECOG PS and the number of metastatic sites) could be identified as prognostic factors, their possible impact on response rate and survival was evaluated by performing a multivariate analysis with a logistic regression model and a Spearman correlation model, respectively.

\section{RESULTS \\ Patient Characteristics}

From March 2001 to August 2003, 66 chemotherapynaive patients were assigned using blocked randomization to both treatment arms (33 patients in each arm). Written informed consent was obtained from all enrolled patients. All patients were assessable for response and toxicity.

Patients' baseline characteristics, which were well balanced between the 2 treatment arms, are summarized in Table 1. Fifty-two patients were men (78.8\%), 14 patients were women $(21.2 \%)$, and the median patient age was 60.5 years. The ECOG PS was 0 in 22 patients (33.3\%), 1 in 42 patients (63.6\%), and 2 in the remaining 2 patients (3\%).

Twenty-nine of 66 patients (43.9\%) presented with $\geq 2$ metastatic sites, with lymph nodes the most frequent disease localization (82\%), followed by bone (27.3\%) and liver (27.3\%). Histologic types were adenocarcinoma in 48 patients $(72.7 \%)$, squamous cell in 7 patients $(10.6 \%)$, anaplastic in 5 patients (7.6\%), epithelioid in 5 patients $(7.6 \%)$ and medullary in 1 patient (1.5\%).

\section{Response}

In total, 297 cycles (155 cycles in the CGT arm; 142 cycles in the CGV arm) were administered with a median of 4 cycles of treatment per patient in the vinorelbine-containing arm and 5 cycles per patient in the paclitaxel-containing arm. According to the research protocol, we proceeded to the second step of the study after obtaining 5 objective responses in the first 18 patients in each treatment arm. In the CGT arm, 16 patients achieved an objective response (48.5\%), 1 patient achieved a CR (3\%) and 15 patients achieved a PR (45.5\%); in the CGV arm, the ORR was $42.3 \%$, and there were 3 CRs $(9 \%)$ and 11 PRs (33.3\%).

Nine patients in the CGT arm and 8 patients in the CGV arm had SD as their best response $(27.3 \%$ and $24.2 \%$, respectively), whereas PD occurred in 8 patients $(24.2 \%)$ in the CGT arm and in 11 patients 
TABLE 1

Patient Characteristics

\begin{tabular}{|c|c|c|c|}
\hline \multirow[b]{2}{*}{ Characteristic } & \multicolumn{3}{|c|}{ No. of patients (\%) } \\
\hline & Total & CGT & CGV \\
\hline Total enrolled & $66(100)$ & $33(50)$ & $33(50)$ \\
\hline Assessable for activity & $66(100)$ & $33(50)$ & $33(50)$ \\
\hline Assessable for toxicity & $66(100)$ & $33(50)$ & $33(50)$ \\
\hline \multicolumn{4}{|l|}{ Age, y } \\
\hline Median & 60.5 & 60 & 61 \\
\hline Range & $31-75$ & $33-75$ & $31-75$ \\
\hline \multicolumn{4}{|l|}{ Sex } \\
\hline Men & $52(78.8)$ & $25(75.5)$ & $27(81.8)$ \\
\hline Women & $14(21.2)$ & $8(24.3)$ & $6(18.2)$ \\
\hline \multicolumn{4}{|l|}{ ECOG PS } \\
\hline 0 & $22(33.3)$ & 10. (30.3) & $12(36.4)$ \\
\hline 1 & $42(63.6)$ & $22(66.7)$ & $20(60.6)$ \\
\hline 2 & $2(3)$ & $1(3)$ & $1(3)$ \\
\hline \multicolumn{4}{|l|}{ Metastatic sites } \\
\hline 1 & $37(56)$ & $19(57.6)$ & $18(54)$ \\
\hline 2 & $16(24)$ & $8(24)$ & $8(24)$ \\
\hline$\geq 3$ & $13(20)$ & $6(18.2)$ & $7(21)$ \\
\hline \multicolumn{4}{|l|}{ Site of disease } \\
\hline Lung & $16(24)$ & $6(18.2)$ & $10(30)$ \\
\hline Lymph nodes & $54(82)$ & $29(87.9)$ & $25(75.7)$ \\
\hline Bone & $18(27.3)$ & $7(21)$ & $11(33.3)$ \\
\hline Liver & $18(27.3)$ & $8(24)$ & $10(30.3)$ \\
\hline Pleura & $6(9.1)$ & $3(9.1)$ & $3(9.1)$ \\
\hline Skin & $1(1.5)$ & $1(3)$ & 0 \\
\hline Pancreas & $3(4.5)$ & $3(9.1)$ & 0 \\
\hline Other & $8(12.1)$ & $3(9.1)$ & $5(15)$ \\
\hline \multicolumn{4}{|l|}{ Histologic type } \\
\hline Adenocarcinoma & $48(72.7)$ & $25(75.7)$ & $23(73)$ \\
\hline Anaplastic carcinoma & $5(7.5)$ & $3(9.1)$ & $2(6.1)$ \\
\hline Squamous cell carcinoma & $7(10.6)$ & $4(12)$ & $3(9.1)$ \\
\hline Medullary carcinoma & $1(1.5)$ & 0 & $1(3)$ \\
\hline Epithelioid carcinoma & $5(7.6)$ & $1(3)$ & 4 (12) \\
\hline
\end{tabular}

C indicates cis-diamminedichloroplatinum; G, gemcitabine; T, paclitaxel; V, vinorelbine; ECOG PS, Eastern Cooperative Oncology Group performance status.

(33.3\%) in the CGV arm (Table 2). The median response duration was similar in the 2 treatment arms: 7.5 months $(95 \%$ confidence interval $[95 \% \mathrm{CI}], 5.6-$ 9.4 months) in the CGV arm (mean, 10.28 months; range, 1-21.4 months) and 7.4 months (95\% CI, 6.98.0 months) in the CGT arm (mean, 9 months; range, 2.2-16.7 months).

The median OS was 13.6 months (95\% CI, 6.6120.59 months; mean, 12.14 months; range, 1.03-21.5 months) for patients who received the CGV combination. For patients who received the CGT combination, the median OS was 9.6 months (95\% CI, 7.1112.09 months; mean, 10 months; range, 1.5-16.73 months). The median TTP also was similar in both treatments arms (6 months). The Kaplan-Meier curves for OS and TTP for each treatment arm are represented in Figures 1 and 2 , respectively.
TABLE 2

Response

\begin{tabular}{lccc}
\hline & \multicolumn{3}{c}{ No. of patients (\%) } \\
\cline { 2 - 4 } $\begin{array}{l}\text { Response (N = 66 } \\
\text { assessable patients) }\end{array}$ & CGT & CGV & $\begin{array}{l}\text { Overall patient } \\
\text { population }\end{array}$ \\
\hline CR & $1(3)$ & $3(9)$ & $4(6)$ \\
PR & $15(45.5)$ & $11(33.3)$ & $26(39.4)$ \\
SD & $9(27.3)$ & $8(24.2)$ & $17(25.8)$ \\
PD & $8(24.2)$ & $11(33.3)$ & $19(28.8)$ \\
\hline
\end{tabular}

$\mathrm{C}$ indicates cis-diamminedichloroplatinum; G, gemcitabine; T, paclitaxel; V, vinorelbine, $\mathrm{CR}$, complete response; $\mathrm{PR}$, partial response; $\mathrm{SD}$, stable disease; $\mathrm{PD}$, progressive disease.

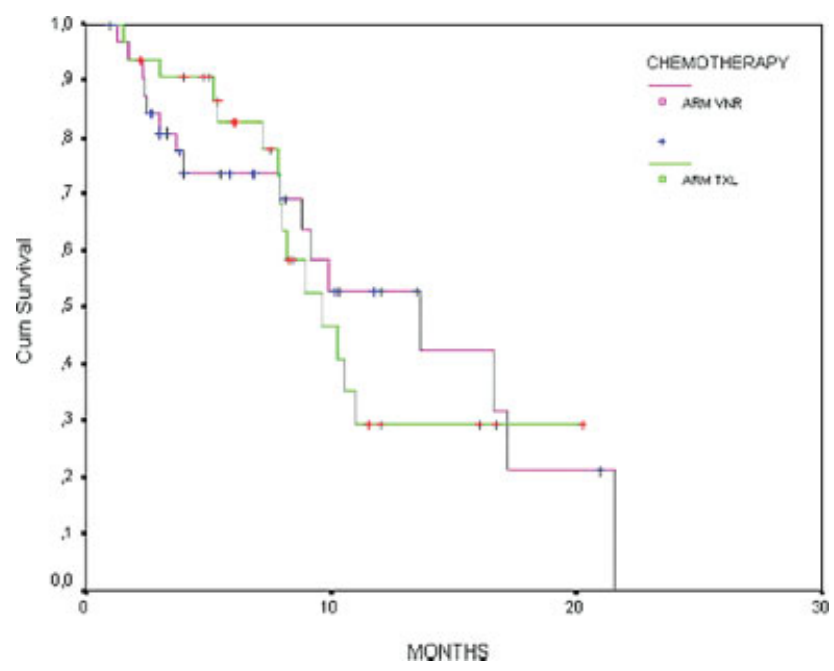

FIGURE 1. Kaplan-Meier estimates of overall survival. VNR indicates vinorelbine; TXL, paclitaxel.

With reference to clinical parameters, such as prognostic factors, no correlation between the response rate and any clinical parameter was observed. However, there was a significant correlation between OS and PS (Spearman $\rho,-0.263$; 2-tailed $P<.05$ ), so that OS decreased with increasing PS.

\section{Compliance With Treatment and Toxicity}

The tolerability of the 2 treatment arms was acceptable. Overall, grade 3 and 4 toxicities were more frequent in the CGT arm, with 16 patients (48.5\%) experiencing grade 3 or 4 adverse events versus 11 patients (33.3\%) in the CGV arm. In the CGT arm, the most common hematologic toxicity was anemia, which occurred in 15 patients (45.5\%) and was grade 3 or 4 in 3 patients (9.1\%). In the CGV arm, the most common hematologic toxicity was neutropenia, which was experienced by 14 patients (42\%) and was grade 3 or 4 in 4 patients (12\%). 


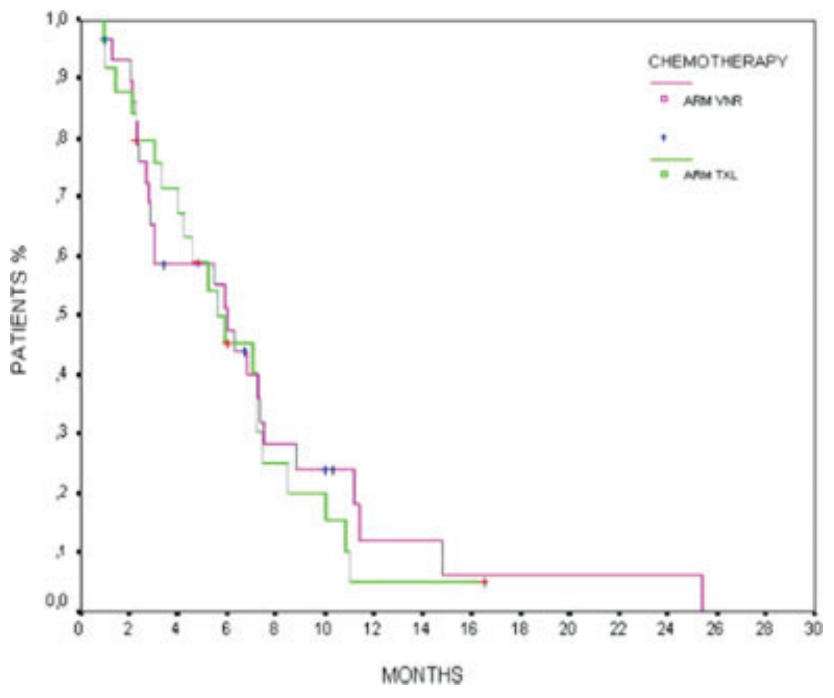

FIGURE 2. Kaplan-Meier estimates of time to progression. VNR indicates vinorelbine; TXL, paclitaxel.

TABLE 3

Hematologic Toxicity (Grades 3 and 4)

\begin{tabular}{llll}
\hline & \multicolumn{3}{c}{ No. of patients (\%) } \\
\cline { 2 - 4 } $\begin{array}{l}\text { Toxicity (N = 66 } \\
\text { assessable patients) }\end{array}$ & CGT & CGV & 0verall patient population \\
\hline Neutropenia & $6(18.2)$ & $4(12)$ & $10(15)$ \\
Anemia & $3(9.1)$ & $3(9.1)^{*}$ & $6(9)$ \\
Thrombocytopenia & $4(12)$ & $3(9.1)$ & $7(10.6)$ \\
\hline
\end{tabular}

C indicates cis-diamminedichloroplatinum; G, gemcitabine; $\mathrm{T}$, paclitaxel; $\mathrm{V}$, vinorelbine. * Grade 3.

No febrile neutropenia was reported. Details about hematologic toxicity are reported in Table 3.

Among nonhematologic toxicities, episodes of nausea and emesis prevailed in both treatment arms (15 grade 1 or 2 episodes; 1 grade 3 or 4 episode), although they were more frequent in the CGT arm (8 grade 1 or 2 episodes; 1 grade 3 episode) than in the CGV arm (7 grade 1 or 2 episodes; 0 grade 3 or 4 episodes). Nonhematologic toxicities are reported in Table 4.

In the paclitaxel-containing arm, there was a single treatment withdrawal (caused by the onset of grade 3 cardiac toxicity), and there were 5 25\%-dose reductions ( 2 caused by thrombocytopenia and 3 caused by renal toxicity, neutropenia, and nausea/ emesis, respectively); whereas, in 2 other patients the scheduled dose for Day $\geq 8$ was omitted (because of anemia and thrombocytopenia, respectively).

In the vinorelbine-containing arm, no treatment withdrawal was observed; whereas there were $725 \%$ dose reductions (caused by thrombocytopenia, diarrhea, stomatitis, renal toxicity, neutropenia, nausea/
TABLE 4

Nonhematologic Toxicity (Grades 3 and 4)

\begin{tabular}{llll}
\hline & \multicolumn{3}{c}{ No. of patients (\%) } \\
\cline { 2 - 4 } $\begin{array}{l}\text { Toxicity (N = 66 } \\
\text { assessable patients) }\end{array}$ & CGT & CGV & 0verall patient population \\
\hline Nausea/emesis & $1(3)^{*}$ & 0 & $1(1.5)$ \\
Diarrhea & 0 & $1(3)^{*}$ & $1(1.5)$ \\
Mucositis & $1(3)^{*}$ & 0 & $1(1.5)$ \\
Neurotoxicity & 0 & 0 & 0 \\
Hepatotoxicity & 0 & 0 & 0 \\
Asthenia & 0 & 0 & 0 \\
Cardiotoxicity & $1(3)^{*}$ & 0 & $1(1.5)$ \\
Nephrotoxicity & 0 & 0 & 0 \\
\hline C indicates cis-diamminedichloroplatinum; G, gemcitabine; T, paclitaxel; V, vinorelbine. \\
*Grade 3.
\end{tabular}

emesis, and anemia in 1 patient each). In 9 patients, the scheduled dose for Day $\geq 8$ was omitted (in 4 patients because of thrombocytopenia, in 3 patients because of neutropenia, and in 2 patients because of nausea/emesis and anemia).

\section{DISCUSSION}

CUPs constitute a heterogeneous and relatively rare group of malignancies. The survival of patients with CUP can vary mainly on the basis of histology, number and sites of disease, PS, and sex. For example, patients who present with neuroendocrine carcinoma or squamous cell carcinoma that infiltrates cervical lymph nodes who have $<2$ sites of disease, and lymph nodes as the only metastatic site, a good PS $(<1)$ and who also are women are characterized by a more favorable prognosis. Conversely, adenocarcinomas and liver metastases are associated with poorer survival. ${ }^{1,10}$

The optimal first-line treatment for patients with CUP remains to be determined. ${ }^{2,5}$ Previous trials of noncisplatin-based combinations, including 5-fluorouracil, cyclophosphamide, and doxorubicin, yielded disappointing results with response rates ranging from $10 \%$ to $33 \%$ and with median survival ranging from 4 months to 9 months. ${ }^{11-17}$ Although cisplatinbased regimens have yielded better response rates (usually in the range of 23-39\%), median survival results remain unsatisfactory, ranging from 5 months to 9 months. ${ }^{14,15,17-19}$

Until the 1990s, only 1 of 3 published Phase III, randomized trials in which a cisplatin-based regimen was compared with a noncisplatin-based regimen reported a survival advantage for patients who received cisplatin. ${ }^{14,15,20}$ However, the suboptimal design of those Phase III studies, including small sample sizes 
TABLE 5

Recent Phase II Studies in Patients with Cancer of Unknown Primary Origin (With Taxane- and Nontaxane-based Chemotherapy)

\begin{tabular}{|c|c|c|c|c|}
\hline Author and year of publication & No. of patients & Regimen & Response rate (\%) & $\begin{array}{l}\text { Median survival } \\
\text { (Months) }\end{array}$ \\
\hline Briasoulis et al, $2000^{21}$ & 77 & $\mathrm{CbT}$ & 38.7 & 13 \\
\hline Greco et al, $2000^{22}$ & 71 & CbET & 48 & 11 \\
\hline \multirow[t]{2}{*}{ Greco et al, $2000^{23}$} & 26 & CDoc & 26 & 8 \\
\hline & 47 (Sequential) & CbDoc & 22 & 8 \\
\hline \multirow[t]{2}{*}{ Dowell et al, $2001^{34}$} & 34 & $\mathrm{CbE}$ & 19 & 8.4 \\
\hline & & TFL & 19 & 6.5 \\
\hline Greco et al, $2002^{24}$ & 120 & CbGT & 25 & 9 \\
\hline Balana et al, $2003^{29}$ & 30 & CEG & 36.6 & 7.2 \\
\hline \multirow[t]{2}{*}{ Culine et al, $2003^{30}$} & 80 & GC & 55 & 8 \\
\hline & & IC & 38 & 6 \\
\hline Greco et al, $2004^{25}$ & 132 & CbET GI & 30 & 9.1 \\
\hline Pouessel et al, $2004^{35}$ & 36 & DocG & 40 & 10 \\
\hline Piga et al, $2004^{31}$ & 102 & CbEA & 26.5 & 9 \\
\hline Park et al, $2004^{36}$ & 37 & $\mathrm{TC}$ & 42 & 11 \\
\hline El-Rayes et al, $2005^{37}$ & 22 & $\mathrm{CbT}$ & 23 & 6.5 \\
\hline Schneider et al, $2005^{38}$ & 27 & CbGCape & 41 & 6.5 \\
\hline \multirow[t]{2}{*}{ Huebner et al, $2005^{32}$} & 92 & CT & 21.6 & 10.7 \\
\hline & & GV & 21.4 & 6.9 \\
\hline Pittman et al, $2005^{39}$ & 50 & $\mathrm{GCb}$ & 30 & 8.1 \\
\hline Varadhachary et al $2005^{40}$ & 16 (5 2nd line) & GOx & 36 & NR \\
\hline \multirow[t]{2}{*}{ Palmeri et al (current study) } & 66 & CGT & 48.5 & 9.6 \\
\hline & & CGV & 42.3 & 13.6 \\
\hline
\end{tabular}

Cb indicates carboplatin; T, paclitaxel; E, etoposide; C, cis-diamminedichloroplatinum; Doc, docetaxel; F, fluorouracil; L, leucovorin; G, gemcitabine; I, irinotecan; A, doxorubicin; Cape, capecitabine; Ox, oxaliplatin; NR, not reported; V, vinorelbine.

(55-95 patients), did not allow the authors to reach definitive conclusions about the role of cisplatin in the treatment of CUP.

Given the lack of randomized Phase III trials, it seemed necessary for us also to focus our attention on published Phase II studies. Indeed, Table 5 lists the more recent and/or important Phase II trials of different chemotherapy regimens in the treatment of CUP and includes the current study. It seems pertinent to remark here that, when reviewing the literature with clinical data based mainly on these kinds of studies, all results must be reviewed with a skeptical eye and comparisons between different Phase II studies may not be accurate.

In the last decade, the availability of new, broadly active compounds, such as taxanes, vinorelbine, gemcitabine, and irinotecan, has represented an important gain in the management of CUP. In a Phase II trial, Briasoulis et $\mathrm{al}^{21}$ evaluated the combination of carboplatin plus paclitaxel in 77 patients with CUP (including 33 patients with liver metastases, bone metastases, or multiorgan metastases). The ORR was $38.7 \%$ (CR rate, 20\%) and the median OS was 13 months in the entire population and 10 months in the poor-prognosis group (visceral or disseminated metas- tases). It is noteworthy that the $68.4 \%$ of patients who presented with peritoneal carcinomatosis may have contributed to the better results. A large group of patients with CUP (396 patients), excluding favorable subsets, was treated with taxane-containing regimens in 5 consecutive Phase II trials by the Minnie Pearl Cancer Research Network. ${ }^{22-25}$ Overall, those 5 studies had an ORR of $30 \%$, a median OS of 9.1 months, a progression-free survival of 5 months, 1-year and 2-year survival rates of $38 \%$ and $19 \%$, respectively. The authors compared their results with historic data from 45 prospective trials (1515 patients) and with retrospective reviews (31,419 patients), which showed that the 1-year and 2-year survival rates were prolonged significantly. ${ }^{26}$

Within the group of new agents, gemcitabine can be considered a good candidate for polychemotherapy regimens in the treatment of CUP because of its wide spectrum of activity and good tolerability profile. Initially, this drug was given as second-line treatment. ${ }^{27}$ More recently, on the basis of these encouraging results published in 2001 and confirmed in 2005, ${ }^{28}$ gemcitabine has been investigated more largely investigated in the first-line setting.

Balana et $\mathrm{al}^{29}$ conducted a Phase II study in 30 patients who had CUP with poor prognostic features 
( $10 \%$ of patients had brain metastases, $30 \%$ of patients had bone metastases and $20 \%$ of patients had liver metastases) to evaluate a regimen of cisplatin, etoposide and gemcitabine every 21 days. Those investigators reported an ORR of $36.6 \%$ and a median survival of 7.21 months with grade 3 or 4 neutropenia in $60 \%$ of patients.

In the study of Culine et al,$^{30} 80$ patients $(60 \%$ with poor prognostic features) were randomized to receive either cisplatin in combination with gemcitabine (GC) or irinotecan (IC). The reported ORR was 55\% in the GC arm and $38 \%$ in the IC arm and the median survival was 8 months and 6 months, respectively, with an unfavorable toxicity profile in the IC group (2 toxic deaths from septic shock).

In 2004, Piga et $\mathrm{al}^{31}$ confirmed response and survival data when using a platinum-containing regimen (carboplatin, doxorubicin, and etoposide) in a Phase II study of 102 patients with CUP. An ORR of $26.5 \%$ and an OS of 9 months (progression-free survival, 4 months) were reported, whereas the main toxicity was grade 3 or 4 hematologic toxicity, which was reported in $57.8 \%$ of patients.

Vinorelbine has shown good activity in patients with lung, breast, head/neck, ovarian and uterine carcinomas and has an acceptable toxicity profile. Few studies have evaluated the role of this drug in the treatment of CUP. Recently, in a randomized Phase II trial results that have been published only in an abstract form, Huebner et $\mathrm{al}^{32}$ treated 92 patients with CUP using either paclitaxel plus carboplatin or gemcitabine plus vinorelbine. In those 2 treatment arms, the ORR was $21.6 \%$ and $21.4 \%$, respectively; the median OS was 10.7 months and 6.9 months, respectively; and the median progression-free survival was 6.4 months and 4 months, respectively.

Because of this background and considering that most CUPs have their origin either in the pancreas or in the lung, ${ }^{33}$ there was a strong rationale for designing a study in which cisplatin and gemcitabine were combined with either paclitaxel or vinorelbine. Overall, in the current study, the patients were a good prognostic group for CUP because of the following characteristics: relatively young age (60 years), good PS ( 0 or 1 for nearly all patients), prevalence of histology with a better prognosis (adenocarcinoma, $72.7 \%$; squamous cell carcinoma, $10.6 \%)$, relatively few patients with liver involvement (27.3\%) and many patients with only 1 metastatic site $(56 \%)$.

In this cohort both combinations, CGT and CGV, satisfied the 2-step design and demonstrated antitumor activity without any relevant differences observed between them in terms of ORR $(48.5 \%$ vs $42.3 \%)$ or response duration (7.5 months vs 7.4 months). How- ever, the CGV arm yielded superior results in terms of both OS (13.6 months vs 9.6 months) and treatment tolerability. Grade 3 and 4 neutropenia was only observed in $18.2 \%$ of patients on the CGT arm and in $12 \%$ of patients on the CGV arm. No febrile neutropenia was observed. Nausea and emesis, which generally were mild, were more frequent in the CGT group than in the CGV group. No toxic deaths were observed.

Our findings confirm that cisplatin, gemcitabine, paclitaxel and vinorelbine are active in patients with CUP. Even though survival is not an endpoint for a Phase II study, the survival results observed for the vinorelbine-containing combination appear to be extremely noteworthy, because they match the best results reported in the literature to date. Furthermore, the good tolerability profile of the vinorelbine-containing combination is a fundamental element to take into account when treating patients with a poor life expectancy, like that for patients with CUP. It must be taken into consideration that, as mentioned above, patient selection can interfere significantly with the results of a randomized Phase II study, depending on the prognostic characteristics of the patients who are selected. In conclusion, through lack of a deeper knowledge of the biologic and molecular characteristics of these particular neoplasms and according to a pick the winner attitude, we believe that the combination of CGV deserves consideration as an experimental arm in the design of future randomized, Phase III trials for patients with CUP.

\section{REFERENCES}

1. Pavlidis N, Fizazi K. Cancer of unknown primary (CUP). Crit Rev Oncol Hematol. 2005;54:243-250.

2. Greco FA, Hainsworth JD. Tumors of unknown origin. $C A$ Cancer J Clin. 1992;42:96-115.

3. Culine S, Kramar A, Saghatchian M, et al. Development and validation of a prognostic model to predict the length of survival in patients with carcinomas of an unknown primary site. J Clin Oncol. 2002;20:4679-4683.

4. Pavlidis N, Kosmidis P, Skarlos D, et al. Subsets of tumors responsive to cisplatin or carboplatin combinations in patients with carcinoma of unknown primary site. A Hellenic Cooperative Oncology Group Study. Ann Oncol. 1992;3:631-634.

5. Bugat R, Bataillard A, Lesimple T, et al. Summary of the standards, options and recommendations for the management of patients with carcinoma of unknown primary site. Br J Cancer. 2003;89(suppl 1):S59-S66.

6. Miller AB, Hoogstraten B, Staquet M, et al. Reporting results of cancer treatment. Cancer. 1981;47:207-214.

7. Trotti A, Colevas AD, Setser A, et al. CTCAE version 3.0: development of a comprehensive grading system for the adverse effects of cancer treatment. Semin Radiat Oncol. 2003;13:176181.

8. Simon R. Optimal two-stage designs for Phase II clinical trials in oncology. Control Clin Trials. 1989;10:1-10.

9. Kaplan EL, Meier P. Non parametric estimation from incomplete observations. J Am Stat Assoc. 1958;53:457-481. 
10. Hess KR, Abbruzzese MC, Lenzi R, Raber MN, Abbruzzese JL. Classification and regression tree analysis of 1000 consecutive patients with unknown primary carcinoma. Clin Cancer Res. 1999;5:3403-3410.

11. Goldberg RM, Smith FP, Ueno W, et al. 5-Fluorouracil, Adriamycin and mitomycin in the treatment of adenocarcinoma of unknown primary. J Clin Oncol. 1986;4:395-399.

12. Treat J, Falchuk SC, Tremblay C, et al. Phase II trial of methotrexate-FAM (m-FAM) in adenocarcinoma of unknown primary. Eur J Clin Oncol. 1989;25:1053-1059.

13. Woods RL, Fox RM, Tattersall MHN, et al. Metastatic adenocarcinomas of unknown primary: a randomized study of two combination-chemotherapy regimens. $N$ Engl J Med. 1980; 303:87-89.

14. Milliken ST, Tattersall MHN, Woods RL, et al. Metastatic adenocarcinoma of unknown primary site: a randomized study of two combination chemotherapy regimens. Eur J Cancer Clin Oncol. 1987;23:1645-1650.

15. Eagan RT, Therneau TM, Rubin J, Long HJ, Schutt AJ. Lack of value for cisplatin added to mitomycin-doxorubicin combination chemotherapy for carcinoma of unknown primary site. A randomized trial. Am J Clin Oncol. 1987;10:82-85.

16. Anderson $\mathrm{H}$, Thatcher N, Randin E, et al. VAC (vincristine, Adriamycin and cyclophosphamide) chemotherapy for metastatic carcinoma from an unknown primary site. Eur J Cancer Clin Oncol. 1983;19:49-52.

17. De Campos ES, Menace LP, Radford J, et al. Metastatic carcinoma of uncertain primary site: a retrospective review of 57 patients treated with vincristine, doxorubicin, cyclophosphamide (VAC) or VAC alternating with cisplatin and etoposide (VAC/EP). Cancer. 1994;73:470-475.

18. Raber MN, Faintuch J, Abbruzzese JL, et al. Continuous infusion 5-fluorouracil, etoposide and cis-diamminedichloroplatinum in patients with metastatic carcinoma of unknown primary origin. Ann Oncol. 1991;2:519-520.

19. Gill I, Guaglianone P, Grunberg SM, et al. High dose intensity of cisplatin and etoposide in adenocarcinoma of unknown primary. Anticancer Res. 1991;11:1231-1235.

20. Falkson CI, Cohen GL. Mitomycin and cisplatin versus mitomycin alone as therapy for carcinoma of unknown primary origin. Oncology. 1998;55:116-121.

21. Briasoulis E, Kalofonos H, Bafaloukos H, et al. Carboplatin plus paclitaxel in unknown primary carcinoma: a Phase II Hellenic Cooperative Oncology Group study. J Clin Oncol. 2000;18:3101-3107.

22. Greco FA, Burris HA, Erland JB, et al. Carcinoma of unknown primary site. Long-term follow up after treatment with paclitaxel, carboplatin and etoposide. Cancer. 2000;89:2655-2660.

23. Greco FA, Erland JB, Morrissey LH, et al. Carcinoma of unknown primary site: Phase II trials with docetaxel plus cisplatin or carboplatin. Ann Oncol. 2000;11:211-215.

24. Greco FA, Burris HA, Litchy S, et al. Gemcitabine, carboplatin, and paclitaxel for patients with carcinoma of unknown primary site: a Minnie Pearl Cancer Research Network study. J Clin Oncol. 2002;20:1651-1656.

25. Greco FA, Rodriguez GI, Shaffer DW, et al. Carcinoma of unknown primary site: sequential treatment with paclitaxel/ carboplatin/etoposide and gemcitabine/irinotecan: a Minnie Pearl Cancer Research Network Phase II trial. Oncologist. 2004;9:644-652.

26. Greco FA, Litchy S, Dannaher C, et al. Carcinoma of unknown primary site with unfavorable characteristics: survival of 396 patients after treatment with five consecutive phase II trials by the Minnie Pearl Cancer Research Network. Proc Am Soc Clin Oncol. 2004;22:14S. Abstract 4186.

27. Hainsworth JD, Burris HA, Calvert SW, et al. Gemcitabine in the second-line therapy of patients with carcinoma of unknown primary site: a Phase II trial of the Minnie Pearl Cancer Research Network. Cancer Invest. 2001;19:335-339.

28. Hainsworth JD, Spigel DR, Raefsky EL, et al. Combination chemotherapy of gemcitabine and irinotecan in patients with previously treated carcinoma of an unknown primary site: a Minnie Pearl Cancer Research Network Phase II trial. Cancer. 2005;104:1992-1997.

29. Balana C, Manzano J-L, Moreno I, et al. A Phase II study of cisplatin, etoposide and gemcitabine in an unfavourable group of patients with carcinoma of unknown primary site. Ann Oncol. 2003;14:1425-1429.

30. Culine S, Lortholary A, Voigt JJ, et al. Cisplatin in combination with either gemcitabine or irinotecan in carcinomas of unknown primary site: results of a randomized Phase II study. Trial for the French Study Group on Carcinomas of Unknown Primary (GEFCAPI 01). J Clin Oncol. 2003;21: 3479-3482.

31. Piga A, Nortilli R, Cetto GL, et al. Carboplatin, doxorubicin and etoposide in the treatment of tumours of unknown primary site. Br J Cancer. 2004;90:1898-1904.

32. Huebner G, Steinbach S, Kohne CH, et al. Paclitaxel (P)/carboplatin $(\mathrm{C})$ versus gemcitabine $(\mathrm{G})$ /vinorelbine $(\mathrm{V})$ in patients with adeno- or undifferentiated carcinoma of unknown primary (CUP) - a randomized prospective Phase II trial. Proc Am Soc Clin Oncol. 2005;23(16S):330s. Abstract 4089.

33. Le Chevalier T, Cvitkovic E, Caille P, et al. Early metastatic cancer of unknown primary origin at presentation. A clinical study of 302 consecutive autopsied patients. Arch Intern Med. 1988;148:2035-2039.

34. Dowell JE, Garrett AM, Shyr Y, Johnson DH, Hande KR. A randomized Phase II trial in patients with carcinoma of unknown primary site. Cancer. 2001;91:592-597.

35. Pouessel D, Culine S, Becht S, et al. Gemcitabine and docetaxel as front-line chemotherapy in patients with carcinoma of an unknown primary site. Cancer. 2003;100:1257-1261.

36. Park YH, Ryoo BY, Choi SJ, Yang SH, Kim HT. A Phase II study of paclitaxel plus cisplatin chemotherapy in an unfavourable group of patients with cancer of unknown primary site. Jpn J Clin Oncol. 2004;34:681-685.

37. El-Rayes BF, Shields AF, Zalupski M, et al. A Phase II study of carboplatin and paclitaxel in adenocarcinoma of unknown primary. Am J Clin Oncol. 2005;28:152-156.

38. Schneider BJ, Muler J, Philip P, et al. A Phase II trial of carboplatin, gemcitabine and capecitabine in patients with carcinoma of unknown primary site (CUP). Proc Am Soc Clin Oncol. 2005;23(16S):330s. Abstract 4090.

39. Pittman KB, Olver IN, Karapetis CS, et al. Multicenter Phase II study of gemcitabine and carboplatin combination therapy for patients with metastatic carcinoma of unknown primary site: final results. Proc Am Soc Clin Oncol. 2005; 23(16S):764s. Abstract 8142.

40. Varadhachary GR, Blais JC, Raber MN, Lenzi P, Hoff PM, Abbruzzese JL. Phase II study to evaluate the efficacy and toxicity of oxaliplatin in combination with gemcitabine (GEMOX) in cancer of unknown primary (CUP). Proc Am Soc Clin Oncol. 2005;23(16S):342s. Abstract 4140. 